\title{
Scabies, Periorbital Cellulitis and Recurrent Skin Abscesses due to Panton-Valentine Leukocidin-Positive Staphylococcus aureus Mimic Hyper IgE Syndrome in an Infant
}

\author{
Renate Krüger, MD, * Leif G. Hanitsch, MD, + Rasmus Leistner, MD, $\$$ Sylke Schneider-Burrus, MD, 9

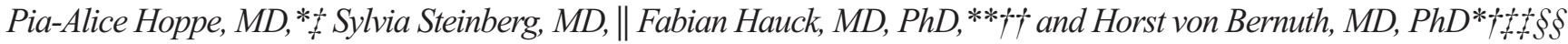

\begin{abstract}
We describe the clinical course of a 2-month-old infant who was evaluated for autosomal dominant Hyper IgE Syndrome based on eczema, periorbital cellulitis, skin abscesses, increased total IgE levels and blood eosinophilia. However, scabies and nasal colonization by Panton-Valentine Leucocidin-positive $S$. aureus were eventually diagnosed. After specific treatment, the child was asymptomatic.
\end{abstract}

Key Words: PVL, S. aureus, scabies, IgE, abscess, hyper IgE syndrome, STAT3

(Pediatr Infect Dis J 2017;36:e347-e348)

$A^{\prime}$ utosomal dominant hyper IgE syndrome (AD-HIES) is a rare genetic disorder characterized by increased total $\operatorname{IgE}$ levels, blood eosinophilia, early onset eczema and recurrent skin, ear and airway infections. Other characteristic findings (e.g., coarse facial features, dental abnormalities, hyperextension of joints) become apparent later in life. The majority of AD-HIES cases are caused by heterozygous dominant-negative mutations in STAT3 (signal transducer and activator of transcription 3). Spontaneous mutations are found in up to $25 \%$ of STAT3 AD-HIES patients. Autosomal recessive HIES - caused by loss-of-function mutations in DOCK8, TYK2 and PGM3 - should be suspected in cases of consanguineous pedigree ( $\mathrm{see}^{1}$ for review).

Scabies is a frequently occurring parasitic skin disease often misdiagnosed as atopic dermatitis. Increased IgE levels in scabies have been described ${ }^{2,3}$

Panton-Valentine Leucocidin-positive Staphylococcus aureus $(\mathrm{PVL}+\mathrm{SA})$ is strongly associated with recurrent deep skin abscesses and soft-tissue infections. ${ }^{4} \mathrm{Nasal} /$ pharyngeal colonization with $\mathrm{PVL}+\mathrm{SA}$ is common in affected patients. Scabies may facilitate $\mathrm{PVL}+\mathrm{SA}$ skin and soft-tissue infections in previously asymptomatic PVL+SA carriers. Increased total IgE levels in Staphlococcus. aureus (S. aureus) infections have been documented. ${ }^{2}$ We report the clinical course of an infant with scabies and nasal PVL+SA colonization that was initially evaluated for AD-HIES.

\section{Accepted for publication February 15, 2017}

From the *Department of Pediatric Pneumology and Immunology, $\downarrow$ Department of Immunology, §Institute of Hygiene and Environmental Medicine, †Sozialpädiatrisches Zentrum, and $\mid$ Department of Dermatology, Charité-Universitätsmedizin Berlin, Berlin, Germany; ||Kinderarztpraxis Invalidenstr., Berlin, Germany; **Department of Pediatric Immunology and Rheumatology, †Immunological Diagnostics Laboratory, Dr. von Hauner Childrens's Hospital, Ludwig-Maximilians-Universität, Munich, Germany; + Labor Berlin GmbH, Fachbereich Immunologie, Charité-Universitätsmedizin -Vivantes, Berlin, Germany; and §§Berlin Center for Regenerative Therapies (BCRT), Charité-Universitätsmedizin Berlin, Berlin, Germany.

The authors have no funding or conflicts of interest to disclose.

Address for correspondence: Renate Krüger, MD, Department of Pediatric Pneumology and Immunology, Charité-Universitätsmedizin Berlin, Augustenburger

Platz 1, 13353 Berlin, Germany. E-mail: r.krueger@charite.de.

Copyright (C) 2017 Wolters Kluwer Health, Inc. All rights reserved.

ISSN: 0891-3668/17/3612-e347

DOI: $10.1097 /$ INF.0000000000001788
A 2-month-old female infant was brought to our outpatient clinic with a history of severe conjunctivitis and periorbital cellulitis with onset of symptoms during the first week of life. The girl had been treated with i.v. ampicillin and gentamicin. S. aureus was cultured from conjunctival swabs. No molecular testing for PVL (polymerase chain reaction for PVL-encoding genes) had been performed. At 5 weeks of age, the infant had developed a pruritic eczema with recurrent pustules/superficial skin abscesses. The girl was the first child of consanguineous Georgian Jews (second-degree cousins) who were both healthy and had no history of atopy, recurrent infections or skin disease. Physical examination of the 2-month-old girl revealed papulous eczema on the trunk and extremities. No other pathological findings were documented.

Laboratory tests showed markedly increased total IgE levels (44.2 kU/1; normal range, 0.0-15). The full blood count showed eosinophilia $(1.65 / \mathrm{nl} ; 18.6 \%)$ white blood cells). Normal results were observed for lymphocyte subpopulations, IgG, IgA, IgM and granulocyte function tests.

A HIES score of 22 (HIES score as proposed in $^{5}$ ) suggested a diagnosis of HIES. Therefore, sequencing of STAT3 was performed. Th17 cells could not be determined initially. The sequencing of STAT3 exon 1-24 and intron-exon boundaries revealed an heterozygous intronic insertion (c.1601-7insT) that had not been previously described. A potential effect on the splicing of exon 18 was proposed and further diagnostic testing (analysis of STAT3 cDNA/protein) was recommended. Before further diagnostic tests, both parents presented with an itchy interdigital skin rash. Scabies was diagnosed. Parents and the infant were treated with permethrin, which resulted in the complete resolution of skin lesions. In light of the history of cellulitis and skin abscesses, nasal swabs were performed on the infant. PVL+SA was detected. Nasal swabs of both parents also showed colonization with PVL + SA. Decolonization measures (as proposed $\left.i^{6}\right)$ were recommended. The child has since been well, and over 12 months of follow-up has showed no further eczema or skin abscesses.

From 2012 to 2015, six patients (aged 6-50 years) without a history of atopy or parasitic infections were referred to our outpatient clinics for evaluation for HIES based on recurrent skin abscesses and increased total IgE levels (616-3203 kU/1). However, no other characteristics of HIES (e.g., early onset eczema, airway infections) were observed in these patients, and no immunological or genetic testing was performed. In all patients, PVL+SA was detected in nasal swabs or abscess material. After antibiotic treatment and decolonization measures were carried out, no further skin infections occurred in any of the patients. Based on our observations, we hypothesize that significantly increased $\operatorname{IgE}$ levels are a common finding in patients with $\mathrm{PVL}+\mathrm{SA}$ infections. However, blood sampling (and a determination of total IgE levels) has not been routinely performed on such subjects. Therefore, data that could confirm our hypothesis are limited.

We conclude that skin infections with $\mathrm{PVL}+\mathrm{SA}$ can be associated with increased total IgE levels. PVL+SA and scabies coinfections can mimic HIES, particularly within the first months of life. In patients with eczema, skin or soft-tissue infections and 
increased IgE levels PVL+SA colonization/infection as well as scabies should be considered before immunologic and genetic testing for HIES.

\section{REFERENCES}

1. Farmand S, Sundin M. Hyper-IgE syndromes: recent advances in pathogenesis, diagnostics and clinical care. Curr Opin Hematol. 2015;22:12-22.

2. Przybilla B, Ring J, Völk M. [Total IgE levels in the serum in dermatologic diseases]. Hautarzt. 1986;37:77-82.
3. Walton SF. The immunology of susceptibility and resistance to scabies. Parasite Immunol. 2010;32:532-540.

4. Shallcross LJ, Fragaszy E, Johnson AM, et al. The role of the PantonValentine leucocidin toxin in staphylococcal disease: a systematic review and meta-analysis. Lancet Infect Dis. 2013;13:43-54.

5. Grimbacher B, Schäffer AA, Holland SM, et al. Genetic linkage of hyperIgE syndrome to chromosome 4. Am J Hum Genet. 1999;65:735-744.

6. Gillet Y, Dumitrescu O, Tristan A, et al. Pragmatic management of PantonValentine leukocidin-associated staphylococcal diseases. Int $J$ Antimicrob Agents. 2012;38:457-464. 